\title{
On Fixed Beamforming in Sectored mmWave Ultra Dense Networks at $73 \mathrm{GHz}$
}

Jasmina McMenamy

Trinity College Dublin, Ireland

Haris Kremo

Trinity College Dublin, Ireland

George A. Ropokis

Trinity College Dublin, Ireland

See next page for additional authors

Follow this and additional works at: https://arrow.tudublin.ie/engscheleart

Part of the Electrical and Computer Engineering Commons

\section{Recommended Citation}

J. McMenamy, H. Kremo, G. A. Ropokis and A. Narbudowicz, "On Fixed Beamforming in Sectored mmWave Ultra Dense Networks at 73GHz," 2018 IEEE 29th Annual International Symposium on Personal, Indoor and Mobile Radio Communications (PIMRC), 2018, pp. 1-7, doi: 10.1109/PIMRC.2018.8580769.

This Conference Paper is brought to you for free and open access by the School of Electrical and Electronic Engineering at ARROW@TU Dublin. It has been accepted for inclusion in Conference papers by an authorized administrator of ARROW@TU Dublin. For more information, please contact arrow.admin@tudublin.ie, aisling.coyne@tudublin.ie,gerard.connolly@tudublin.ie. 


\section{Authors}

Jasmina McMenamy, Haris Kremo, George A. Ropokis, and Adam Narbudowicz

This conference paper is available at ARROW@TU Dublin: https://arrow.tudublin.ie/engscheleart/351 


\title{
On Fixed Beamforming in Sectored mmWave Ultra Dense Networks at $73 \mathrm{GHz}$
}

\author{
Jasmina McMenamy*, Haris Kremo*, George A. Ropokis* and Adam Narbudowicz ${ }^{\dagger}$ \\ *CONNECT, Trinity College Dublin \\ \{jmcmena,kremoh,ropokisg\}@tcd.ie \\ ${ }^{\dagger}$ CONNECT, School of Electrical \& Electronic Engineering, Dublin Institute of Technology \\ $\{$ adam.narbudowicz\}@dit.ie
}

\begin{abstract}
We investigate the use of fixed analogue transmit beamforming for downlink communication in millimetre-wave (mmWave) band ultra-dense networks (UDNs) at $73 \mathrm{GHz}$. Our simulation study is based on 3D radiation patterns using a real design of large planar antenna arrays with different horizontal beamwidths and full characterisation of interference. The effects of network densification are captured through different ratios of the number of access points (APs) to the number of users. We quantify the system's performance by evaluating outage and achievable rate. The results show that users with rates around the mean benefit more from the densification than from narrowing the beamwidth, but for users with low rates, narrow beams also provide a significant increase in throughput.
\end{abstract}

\section{INTRODUCTION}

Reducing cell size to improve network capacity has been extensively used in the previous generations of cellular networks. Undoubtedly, this trend of densification of access points (APs) is expected to continue with the network evolution to $5 \mathrm{G}$ and beyond [1]-[3]. With the emphasis on providing immense capacity in hot-spots, the densification will be centred around Ultra-Dense Networks (UDNs) with low power nodes, characterised by short inter-site distance, catering mostly for lowmobility scenarios [4].

Pivotal to the densification of future networks will be millimetre-wave (mmWave) spectrum, formally defined as the frequency range between 30 and $300 \mathrm{GHz}^{1}$. mmWave spectrum offers large bandwidths required to support low latency and very high bit rate applications of $5 \mathrm{G}$ and beyond. Unlike the sub- $6 \mathrm{GHz}$ frequency bands, the mmWave frequency bands provide challenging, but also attractive propagation characteristics when it comes to UDNs. Namely, the mmWave communications channel is characterised by increased path losses and is highly sensitive to blockages, and often consists of only a few communication paths. While these effects introduce several challenges when it comes to reliable communications, they allow for higher spatial frequency reuse, which is a fundamental principle of

\footnotetext{
${ }^{1}$ Industry often uses term mmWave band to denote frequencies between $10 \mathrm{GHz}$ and $100 \mathrm{GHz}$, whereas frequencies between 100 and $300 \mathrm{GHz}$ are referred to as "sub-terahertz" [5].
}

UDNs. Furthermore, highly directional transmission, which is one of the main features of the mmWave systems, compensates for the significant propagation and penetration losses, and further aids higher spatial frequency reuse.

Directionality in mmWave will be achieved through beamforming since at these frequencies, wavelengths provide small spacing between antenna elements. This small spacing then facilitates transceiver architectures with large antenna arrays, which can create a desired radiation pattern that increases antenna gain for the best path and minimises interference for all other directions. However, these radiation patterns are complex, with numerous side-lobes and nulls scattered across all directions. The radiation patterns are obtained by applying adjusted phase and amplitude excitations at each antenna element, eliminating the need for mechanical movement [6]. The adjustments can be realised in analogue or a digital domain. Analogue beamforming, normally implemented using phase shifters, has limited flexibility in dynamically controlling the radiation pattern. Despite this, analogue beamforming is attractive mostly for its simplicity in terms of the number of the required RF chains [7]. On the other hand, digital beamforming relies on the active components that allow fast readjustment of phase and amplitude. However, digital beamforming at mmWave frequencies is prohibitive in terms of complexity, power consumption and cost in general, as it requires a large number of RF chains (1 dedicated RF chain per antenna element) [7]. For these reasons, currently proposed mmWave solutions focus on hybrid configurations, which split beamforming into digital and analogue domains, where an analogue beamformer typically consists of a number of sub-arrays, where each sub-array has a dedicated RF chain.

Considering that mmWave and UDNs - key technologies for future wireless communication, both present challenges in their own right, a deeper understanding of the realistic mmWave UDN deployments is needed. The deployment considerations should include aspects ranging from actual antenna patterns and complexity of hardware architectures to those reflecting the expected 
distribution of users and APs. In that regard, the paper intends to contribute to the state-of-the-art by providing the antenna design for sectored APs at $73 \mathrm{GHz}$ and detailed performance analysis of the mmWave UDNs with clustered user distribution and different densities of APs. We consider simple beamforming architectures - transmit beamforming and employ well established specialised tool (i.e. CST Microwave Studio [8]) to obtain realistic 3D radiation patterns for different horizontal beamwidths of $3^{\circ}, 6^{\circ}, 10^{\circ}$ and $15^{\circ}$, and vertical beamwidth of $10^{\circ}$. Each AP provides all-around coverage with four two-dimensional arrays steerable in the azimuthal plane.

\section{A. Related work \& main contributions}

A body of work has started to emerge on systemlevel performance aspects of mmWave cellular systems. Some focus on analytical approaches, such as the work in [7], which provides a comprehensive overview of the analytical techniques as well as state-of-the-art on mmWave and key considerations in the design of mmWave systems. A notable numerical and experimental analysis is given in [9], where the authors provide a detailed statistical mmWave channel model and parameters for $28 \mathrm{GHz}$ and $73 \mathrm{GHz}$, obtained using highly directional horn antennas. The system capacity analysis incorporates transmitter and receiver beamforming gains, which are derived based on the experimentally obtained channel model, assuming specific array configurations. A numerical case study in [10] analyses downlink performance aspects of the $73 \mathrm{GHz}$ mmWave network with the increasing density of base stations. The study uses analytically-obtained antenna patterns, assuming specific array configurations on the transmitter and receiver side. It also compares mmWave network performance with the $2 \mathrm{GHz}$ microwave omnidirectional network. A comprehensive analysis of UDNs with different small cells densities, where inter-site distances range from 200 to 5 meters is given in [3]. The study is done for different user densities, and for the sub-6GHz and $10 \mathrm{GHz}$ frequencies. In terms of beamforming, the work considers digital beamforming (in the horizontal plane) and $4 \times 4$ antenna elements. In [11], the authors, amongst others, analyse system-level aspects of analogue beamforming with analytically synthesised antenna patterns in a mmWave network with 3-sector sites, taking into account imperfect channel feedback, as well as the impact of some hardware impairments.

Recent work in [12] pursues a similar objective to our work in considering the implications of realistic antenna patterns on the system-level performance in mmWave networks. There, the antenna arrays are used at the transmitter and receiver, with the base station array covering $120^{\circ}$ sector. The array radiation pattern uses simulated single patch antenna, while the array factor is based on the analytical expression. The work focuses on the SINR distributions. Our work, on the other hand, addresses fixed analogue transmit beamforming on the downlink, with the designed antenna array at $73 \mathrm{GHz}$ and $3 \mathrm{D}$ radiation patterns that capture a range of horizontal beamwidths. Furthermore, system level simulations focus on the rate aspects of the performance, taking into account the implications of a single and multi-beam transmission per a sector/array.

In summary, our work advances the antenna design and current mmWave system-level studies by providing the following main contributions:

1) $3 \mathrm{D}$ antenna design at $73 \mathrm{GHz}$ for sectored APs, with horizontal beamwidths of $3^{\circ}, 6^{\circ}, 10^{\circ}$ and $15^{\circ}$, and vertical beamwidth of $10^{\circ}$.

2) Detailed analysis of mmWave UDNs with fixed transmit beamforming, which incorporates the exact 3D antenna patterns and UDN scenarios that feature different density of APs and clustered user distribution.

3) A comparison of the beamforming architecture complexity effects on the user rate. The baseline scenario with one RF chain per a sector/array is compared with a more complex architecture with multi-beam transmission, serving a number of users at a time.

\section{System Model}

This section describes the key components of our system model, starting with a brief description of the adopted mmWave channel model. The section then describes the overall scenario, including the simulation setup. Finally, we present and discuss the radiation patterns.

\section{A. mmWave Channel Model}

We adopt a channel model accounting for free space losses, as well as for three propagation states, namely Line-of-Sight(LoS), Non-Line-of-Sight(NLoS) and outage [9]. For clarity, in this paper, the outage state within the channel model is referred to as out-ofrange state. For a given placement of the transmitter and the receiver, assuming that no out-of-range state occurs, path-loss is modelled as:

$$
P L[d]=\alpha+10 \beta \log _{10}(d)+\xi[d B],
$$

where $\xi \sim \mathcal{N}\left(0, \sigma^{2}\right)$ with $d$ being the distance between the transmitter and the receiver, measured in meters. For a given frequency, parameters $\alpha, \beta$, and $\sigma^{2}$ vary depending on the existence of a LoS path. On the other hand, in case of out-of-range state, path-loss equals to infinity.

Given the above mentioned three states, for the full characterisation of the channel, the knowledge of the probability of the occurrence for each state is required. 
For distance $d$ between the transmitter and the receiver, these probabilities are computed as:

$$
\begin{aligned}
& p_{\text {out }}(d)=\max \left(0,1-\exp \left(-a_{\text {out }} d+b_{\text {out }}\right)\right), \\
& p_{\text {LoS }}(d)=\left(1-p_{\text {out }}(d)\right) \exp \left(-a_{\text {los }} d\right), \\
& p_{N L o S}(d)=1-p_{\text {out }}(d)-p_{\text {LoS }}(d) .
\end{aligned}
$$

Values for parameters $a_{\text {los }}, a_{\text {out }}, b_{\text {out }}$ can be found in [9].

\section{B. Overall setup}

Our scenario considers deployments with different ratios between the number of mmWave APs and active users. While the focus of the analysis is on UDNs, where the AP/user density ratio is expected to be greater or equal to 1 , we also depict the cases where there are $2 \times$ and $3 \times$ more users than mmWave APs. The inclusion of these cases is done to aid better understanding of the impact of the density of mmWave APs with real antenna patterns.

Furthermore, our deployment scenario features clustered distribution of users and distribution of mmWave APs that has constraints on the minimum inter-site distance. Namely, the mmWave APs are randomly distributed in an area similar to Fig. 1, with the constraints on the minimum distance between the nodes as outlined in [13]. Half of the total number of users are clustered around APs - i.e. they are dropped within a certain distance from APs (40m), as specified in [13]. The remainder of users is randomly distributed over the simulation area, although a user may still be dropped close to an AP. Such a configuration, therefore, reflects a typical user distribution and deployment of APs in dense urban areas.

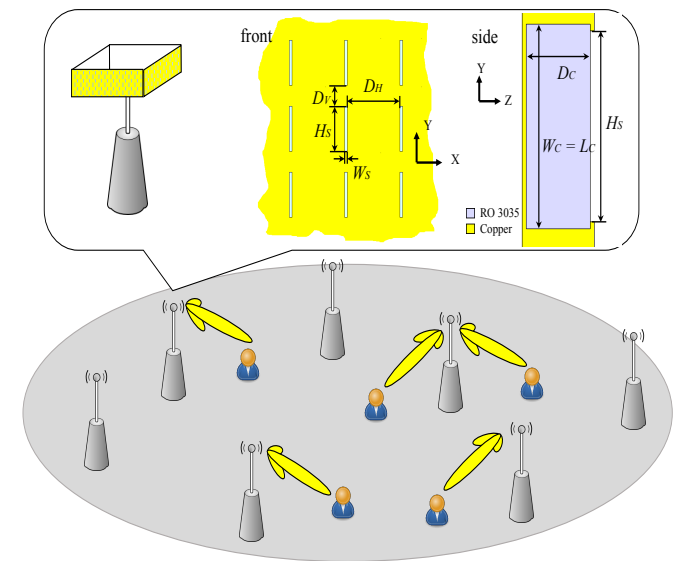

Fig. 1. Deployment scenario with different ratios of AP/user density. Each AP houses four rectangular arrays. The picture also depicts the front and side view of the antenna.

The coverage area of a mmWave AP is sectored using four $N \times M$ planar arrays so that each sector/array covers $90^{\circ}$ of azimuth. The arrays are steerable only in the azimuthal plane.

A single AP serves a randomly distributed user. The received power for user $i$ from $\mathrm{AP} j$, given sector/array $k$, and the corresponding beam $l$ that covers the user is calculated as:

$$
P_{r_{i, j}}^{k, l}=P_{t x}-P L_{i, j}+G_{t x}\left(\phi_{i, j}^{k, l}, \theta_{i, j}^{k, l}\right)+G_{r_{i}}
$$

Here, $P_{t x}$ is the fixed transmit power of the antenna beam (in $\mathrm{dBm}$ ), and $P L_{i, j}$ is distance-dependent pathloss between user $i$ and AP $j$ (in $\mathrm{dB}$ ). $G_{t x}\left(\phi_{i, j}^{k, l}, \theta_{i, j}^{k, l}\right)$ is the transmit beamforming gain (in $\mathrm{dBi}$ ) that depends on the user's azimuth and elevation, with respect to sector $k$, computed using the radiation pattern of beam $l . G_{r_{i}}$ is the receiver antenna gain (in $\mathrm{dBi}$ ) for user $i$, which is a constant in this work. Hence, for each user, the combination of an AP, sector and a beam that provides the maximum received power is selected. Once such a combination is selected, we compute interference from all other (active) beams/APs. Besides path-loss, this computation requires calculating beamforming gain for the user's azimuth and elevation angles to the interfering APs, using the radiation pattern of the interfering beam. Since we use elaborate antenna patterns, it is possible to determine the interference levels quite precisely.

The baseline scenario in our analysis considers a single RF chain per a sector/array, whereby an array, at any point in time, radiates a single beam, serving a single user. In other words, each side of an AP houses antenna array that provides a single fixed beam steered towards a particular angle. If two or more users share the same sector/array, the assumption is that they are time-multiplexed and each user is allocated an equal share of the downlink, in time. Hence, a random user $i$ experiences data rate:

$$
R_{i}=\eta_{i} \cdot B \cdot S E_{i}
$$

where $\eta \in(0,1]$ reflects the effects of this timepartitioning, $B$ is the available bandwidth and $S E_{i}$ is the spectral efficiency of user $i$. For a user within a shared sector/array, factor $\eta$ reflects scheduling of beams in the time domain and is, therefore, inversely proportional to the number of unique beams that cover users within that sector/array. For example, if there are $K$ users that share a sector/array, where each user falls under the coverage of a distinct beam, for each user $\eta=1 / K$. If users are in such proximity that they also share the same beam, for these users, factor $\eta$ is further reduced as a result of employing intra-beam time-domain scheduling, which ensures orthogonality of multiple access to the beam.

Spectral efficiency is calculated to account for the finite code-length and modulation, according to [9] and 
$[14]^{2}:$

$$
S E=\min \left[\log _{2}\left(1+10^{0.1 \times(S N R-\Delta)}\right), S E_{\max }\right]
$$

where $S E_{\max }$ is the maximum spectral efficiency, and $\Delta$ is a loss factor.

Finally, outage, also analysed in this paper, describes the fraction of users (averaged over space in this case) that will achieve a $S I N R \leq T$, where $T$ is some predefined threshold [7]. Hence, all users whose channel state is out-of-range (for all APs) will be a part of outage statistics, as well as users whose SINR (due to noise and/or interference) does not reach the threshold required by the receiver.

1) Simulation setup: Table I outlines the main parameters used in our Matlab-based simulations. The mmWave APs are randomly distributed in a $500 \mathrm{~m} \times 500 \mathrm{~m}$ square area, with 75 active users, which equates to 300 active users $/ \mathrm{km} 2$, similar to the assumptions in [3]. Simulations are averaged over 1000 snapshots, where each snapshot represent a random realisation of distribution of users and APs.

Constraints on the minimum distance between the APs, as well as on the distance between the APs and the users, are according to Table I. Furthermore, as mentioned earlier, half of the users are clustered around APs.

TABLE I

SIMULATION SETUP

\begin{tabular}{|l|l|}
\hline \hline Operating frequency & $73 \mathrm{GHz}$ \\
\hline Bandwidth (B) & $1 \mathrm{GHz}$ \\
\hline $\begin{array}{l}\text { Maximum spectral efficiency } \\
\text { \& spectral efficiency loss }\end{array}$ & $\begin{array}{l}S E_{\max }=4.8 \mathrm{~b} / \mathrm{s} / \mathrm{Hz} \\
\& \Delta=3 \mathrm{~dB}([9])\end{array}$ \\
\hline \hline \# active users & $300 / \mathrm{km} 2$ \\
\hline \#APs / \# users ratio & $0.3,0.5,1,1.5$ \\
\hline Min user distance to an AP & $10 \mathrm{~m}$ \\
\hline Min AP to AP distance & $40 \mathrm{~m}$ \\
\hline AP \& UE height & $10 \mathrm{~m} \mathrm{\&} 1.5 \mathrm{~m}$ \\
\hline \hline $\begin{array}{l}\text { Half-power beamwidth } \\
\text { (HPBW) - azimuth }\end{array}$ & $3^{\circ}, 6^{\circ}, 10^{\circ}, 15^{\circ}$ \\
\hline $\begin{array}{l}\text { Half-power beamwidth } \\
\text { (HPBW) - elevation }\end{array}$ & $10^{\circ}$ \\
\hline \hline Tx power (beam) & $30 \mathrm{dBm}$ \\
\hline Rx gain & $0 \mathrm{dBi}$ \\
\hline \hline
\end{tabular}

\section{Radiation patterns}

The antennas were designed using CST Microwave Studio software [8] and are depicted in Fig. 1. The CST Microwave Studio is a state-of-the-art full-wave electromagnetic simulator utilizing Finite Integration Technique. The software has a high-reliability record and is routinely used for design of industrial antennas.

\footnotetext{
${ }^{2}$ The authors in [14] use the modified Shannon capacity formula that captures losses due to the finite code-length and modulation, and bandwidth efficiency. In this paper, we do not consider bandwidth efficiency, and we use the SE formula as in [9].
}

The antennas are slot antenna arrays, implemented in a Rogers RO3035 substrate of $0.62 \mathrm{~mm}$ height (relative permittivity $\epsilon_{r}=3.5$ and loss coefficient $\delta=0.0015$ ). The front of the board consists of a copper layer, in which a grid of narrow slots with width $W_{S}=0.1 \mathrm{~mm}$ and height $H_{s}=1.4 \mathrm{~mm}$ was etched, as demonstrated in Fig. 1. Distances between edges of neighbouring slots in horizontal and vertical directions are respectively $D_{H}=1.96 \mathrm{~mm}$ and $D_{V}=0.65 \mathrm{~mm}$. This corresponds to the phase centres being separated by half-wavelength at $73 \mathrm{GHz}$. Each slot is backed by a cavity in the RO3035 dielectric substrate of width and length $W_{c}=$ $L_{c}=1.5 \mathrm{~mm}$ and depth $D_{c}=0.55 \mathrm{~mm}$. The cavities simulate antenna feed network implemented in Substrate Integrated Wave-guide (SIW) technology. This technology is commonly used for mmWave frequencies, as it offers the benefit of low-cost and flexibility. In the horizontal plane, the half-power beamwidth varies with the number of elements in the array and may have $15^{\circ}, 10^{\circ}, 6^{\circ}$ or $3^{\circ}$ half-power beamwidth for arrays that are respectively 8 by $7,10,18$ or 34 elements. The maximum realised gain also increases with the number of elements in the array and is respectively: 20.8, 22.3, 24.8 and $27.6 \mathrm{dBi}$. For each array, a sufficient number of beams is generated to cover $90^{\circ}$ sector with half-power beamwidths. The side-lobes in the horizontal plane are approximately $13 \mathrm{~dB}$ below the main beam maxima, which is typical for antenna arrays with uniform power excitation. Fig. 2 shows the exemplary radiation patterns of an array with $15^{\circ}$ half-power beamwidth ( 8 by 7 elements array), which is representative of all four AP sectors/arrays. In the azimuthal plane, the beams are symmetric around $0^{\circ}$ of each array, as depicted by the solid and dashed lines on the left-hand side of Fig. 2. In the elevation plane, depicted on the right-hand side of Fig. 2, every radiation pattern exhibits main beam with $15^{\circ}$ half-power beamwidth, that is tilted $10^{\circ}$ below the horizon. It can be observed that beams directed further away from azimuth angle $\phi=0^{\circ}$ (i.e. vector normal to the plane of the array) exhibit greater side-lobes in the elevation plane, which is the consequence of using flat antenna array panel. All patterns used in this paper were generated by simulated electromagnetic fields in the above-described structure (CST Microwave Studio).

\section{RESULTS}

The focus of this paper is the analysis of the effects that the AP/user density ratio and the antenna patterns, corresponding to a particular beamwidth, have on the average user outage and rate. We start with the outage performance, depicted in Fig. 3. Lower AP/user density ratios of 0.3 and 0.5 are also considered to demonstrate the improvement that UDNs carry. A notable difference in outage can be observed between the case of AP/user density of 0.3 and all other cases, which provide coverage for more than $95 \%$ of users. High AP/user 


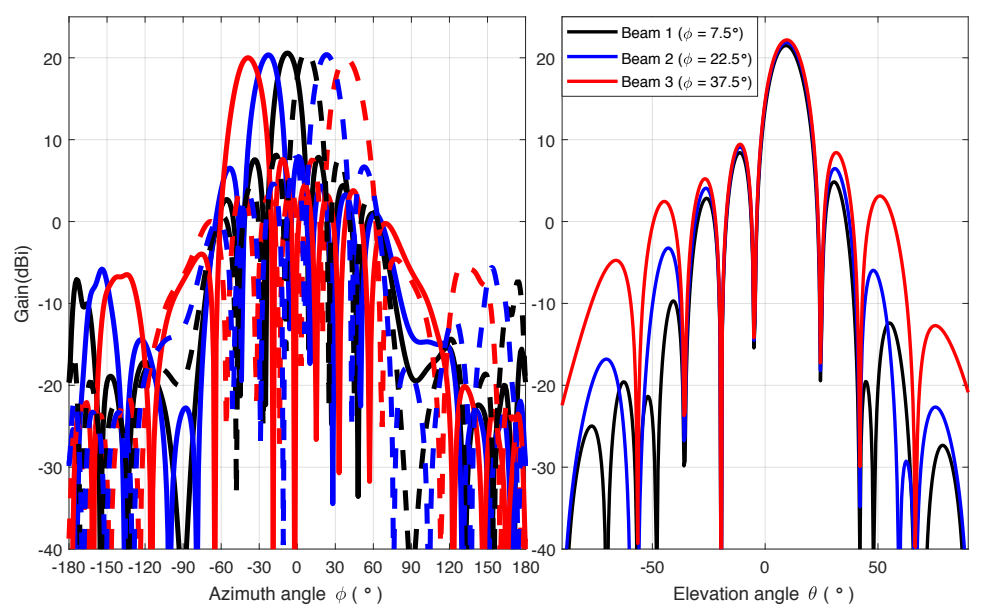

Fig. 2. Horizontal and vertical radiation pattern for $15^{\circ} \mathrm{HPBW}$ (azimuth).

density ratios of 1 and 1.5 practically remove outage, regardless of the beamwidth, as almost all users are within the line of sight of a serving cell. Furthermore, out-of-range state within the channel model, introduced for mmWave links according to Eq. (2), helps significantly in subsiding interference. Namely, we found that over $70 \%$ of non-serving nodes have infinite pathloss. In addition, the directionality of communication also aids SINR, particularly in the case when network deployment is not so dense, such as with 0.3 and $0.5 \mathrm{AP} / \mathrm{user}$ density ratios. In these cases, there is a significant difference in outage performance between, e.g. $3^{\circ}$ and $15^{\circ} \mathrm{HPBW}$. Fig. 4 depicts user rate CDFs

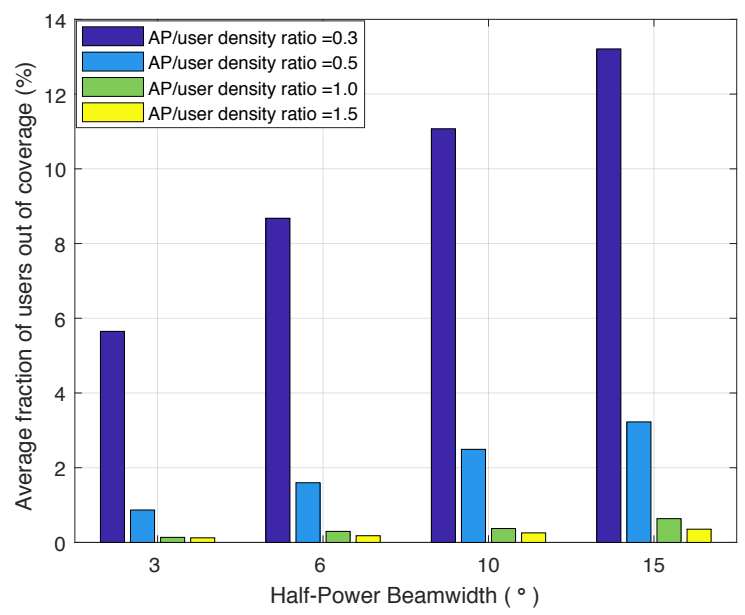

Fig. 3. Outage statistics for different cell/user density ratios and azimuth HPBWs. The threshold value of $0 \mathrm{~dB}$ is used to calculate the outage.

for different beamwidths and AP/user density ratio of 1 . A few trends can be observed from the plot. Firstly, for all beamwidths, the maximum rate is limited to $4.8 \mathrm{~Gb} / \mathrm{s}$ due to the limit on the achievable spectral efficiency according to Eq. (5) and the available bandwidth of
$1 \mathrm{GHz}$. With HPBW of $3^{\circ}$, the probability that a random user will experience a maximum achievable rate is 0.68 , whereas, with HPBW of $15^{\circ}$, this value drops to 0.41 . Hence, the narrow beamwidth of $3^{\circ}$ significantly increases the probability that a user will achieve SINR that will provide the best possible spectral efficiency, according to Eq. (5), compared to the case of HPBW of $15^{\circ}$. We can also see that there is no difference in the median rate between different beamwidths, apart from the $15^{\circ} \mathrm{HPBW}$ case. The situation is different for the users experiencing the lowest rates. For HPBW of $3^{\circ}$, the 5 th-percentile user rate is $1.6 \mathrm{~Gb} / \mathrm{s}$, which drops to $1.08 \mathrm{~Gb} / \mathrm{s}$ with $10^{\circ}$ and $870 \mathrm{Mbps}$ for $15^{\circ}$. Finally, an important trend that can be observed for all beamwidths is the appearance of discontinuities or steps in the CDF. They appear across a range of user rates as a result of two factors: i) maximum spectral efficiency and ii) $\eta$ factor, which accounts for a single-beam transmission per a sector/array and intra-beam scheduling, which is applied when users fall under the coverage of the same beam. Namely, if each sector/array were serving a single user, $\eta=1$. In that case, the users with maximum spectral efficiency would experience maximum rate of $4.8 \mathrm{~Gb} / \mathrm{s}$. However, with users sharing sectors/arrays, factor $\eta$ is reduced (its denominator becomes higher than 1), reducing their rate accordingly. This is the reason the discontinuities appear at a fraction of the maximum rate, i.e. at $2.4 \mathrm{~Gb} / \mathrm{s}, 1.6 \mathrm{~Gb} / \mathrm{s}, 1.2 \mathrm{~Gb} / \mathrm{s}$, etc.

Fig. 5 depicts the mean and 5(\%)-ile user rates for the considered density of APs and beamwidths. In both cases, the AP density is the most significant factor that impacts the results. For example, the improvement in the mean user rate (Fig. 5a) for 3 degrees HPBW is $26 \%$ when AP/user density ratio is increased from 0.5 to 1.5 . For 15 degrees HPBW, this is even more prominent - the improvement is $34.8 \%$. The same trend of a significant increase in the mean user rate is seen when AP/user 


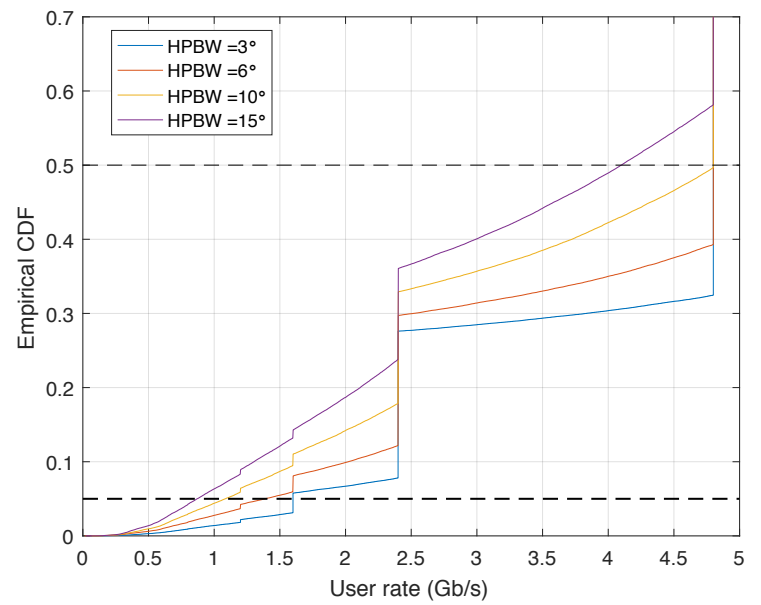

Fig. 4. User rate $\mathrm{CDF}$ for AP/user density ratio of 1 and different azimuth HPBWs

density ratio increases from 0.5 to 1 , again with wider beams seeing somewhat better throughput. However, increasing AP/user density ratio from 1 to 1.5 not only does not provide such significant gains for the mean user rate, but the difference in the improvement between different beamwidths is negligible. For the users with
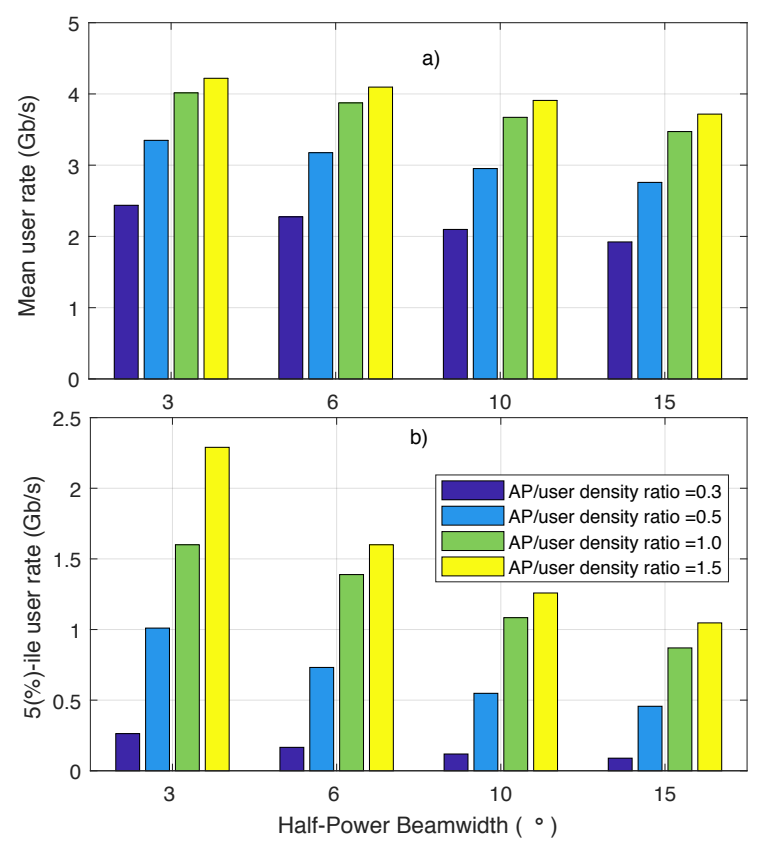

Fig. 5. Mean and 5(\%)-ile user rates for different cell/user density ratios and azimuth HPBWs

the lowest rates (Fig. 5b), large increases of rates can be observed for most cases when AP/user density ratio is increased. Another notable trend is that the impact of beamwidth is also more pronounced compared to the mean user rate. Increasing AP/user density ratio from
0.5 to 1 results in increases in user rate from $58 \%\left(3^{\circ}\right.$ $\mathrm{HPBW})$ to $98 \%\left(10^{\circ} \mathrm{HPBW}\right)$. Different from the mean user rate, here when increasing AP/user density ratio from 1 to 1.5 , there is still a solid improvement to the rate for most beamwidths (between $15 \%$ and $20 \%$ ), while $3^{\circ} \mathrm{HPBW}$ sees over $43 \%$ improvement.

Finally, in scenarios similar to the one considered in this paper, particular attention deserves the impact of APs beamforming architectures, particularly the implications of enabling multi-beam transmissions in each sector. As outlined in Section II-B, our analysis is focused on the baseline scenario with a single-beam transmission, where a single user is served at a time. If, on the other hand, APs are enhanced with multiple RF chains to support multi-beam transmission in each AP sector, there will be no requirement for scheduling of user transmissions, regardless of their positions. Of course, such a case will result in an increased interference for closely spaced users if no other measures are taken for cancellation of interference (such as, e.g. receive beamforming). Nevertheless, factor $\eta$ will take on value 1 for all users, and the rate will only be capped by the maximum achievable spectral efficiency and the bandwidth. Fig. 6a depicts the improvement in the median rate provided by such an enhanced AP configuration with multi-beam (non-orthogonal) transmission for the case of $6^{\circ}$. A considerable increase can be seen in the median throughput for low AP/user density ratios, due to the impact of $\eta$. However, there is no difference between the two cases for the high density of APs. Fig. 6b provides user rate distribution for AP/user density ratio of 1 for both cases. Note a degradation in performance of this enhanced AP, supporting multi-beam transmission, compared to the baseline scenario for the 7 th percentile user rate. This comes as result of interference within the same AP sector. However, between 7 th and 40 th percentile, multibeam even with non-orthogonal transmission provides much better performance than the baseline scenario.
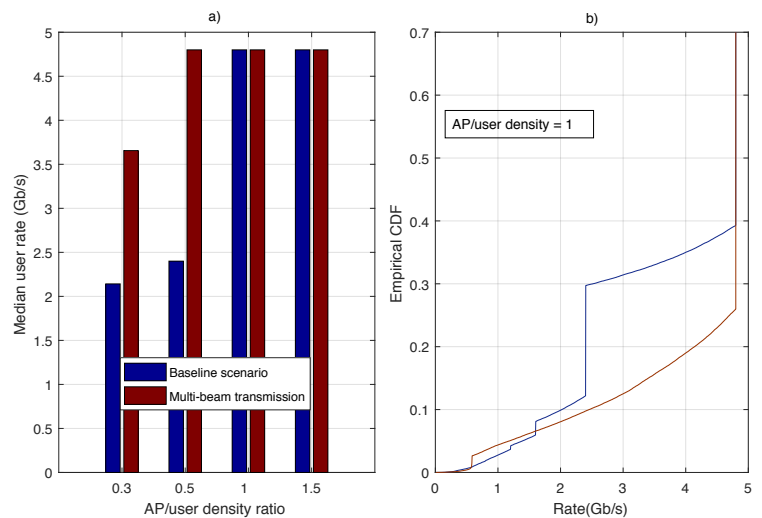

Fig. 6. Comparison between the baseline scenario and the multi-beam transmission - the case of HPBW of $6^{\circ}$ is shown 


\section{CONCLUSION}

This paper addresses performance aspects of mmWave UDNs with analogue transmit beamforming, which due to numerous practical limitations, may be the most optimum choice for downlink data delivery at least in the initial mmWave UDN deployments. We performed extensive computer simulations in the $73 \mathrm{GHz}$ band, using realistic propagation model available in the literature, and most importantly, the elaborate representation of beams obtained through real planar antenna array design. The performance metrics included empirical outage rates and achievable bit-rates, with respect to different AP/user density ratios and halfpower beamwidths. Our results show that the ratio of the number of APs to the number of users of one provides a breaking point since it is the ratio for which there is a significant reduction in the occurrence of users in the complete outage. With the AP density that exceeds the user density, the change in the outage rate is negligible and does not show substantial dependence on the beamwidth. As for the user rate, as expected, the AP density is the dominant factor. Nevertheless, for the same AP density, it is notable that users with lower rates also benefit significantly from narrower beams. For instance, if beamwidth is reduced from $15^{\circ}$ to $3^{\circ}$, the $5 \%$-ile rate increases by more than $80 \%$ for AP/user density ratio of 1 , while for other considered ratios, the improvement is even more significant. We also compared the rate of our baseline single-beam transmission scenario with the case of multiple RF chains that support multi-beam, non-orthogonal transmissions. In general, when these multiple beams are sufficiently spatially separated, interference levels are low, and the more complex, latter architecture provides better throughput. However, for a small number of users located in the proximity of each other, beamwidth as narrow as $3^{\circ}$ is not sufficient for adequate spatial filtration. Then, single-beam transmission and simple time-sharing of the RF chain provides better throughput.

\section{ACKNOWLEDGEMENT}

This publication has emanated from research conducted with the financial support of Science Foundation Ireland (SFI) and is co-funded under the European Regional Development Fund under Grant Number 13/RC/2077 and the EUs Horizon 2020 research and innovation programme under the Marie SkodowskaCurie grant agreement No 713567.

\section{REFERENCES}

[1] J. G. Andrews, S. Buzzi, W. Choi, S. V. Hanly, A. Lozano, A. C. K. Soong, and J. C. Zhang, "What will 5G be?" IEEE Journal on Selected Areas in Communications, vol. 32, no. 6, pp. 1065-1082, June 2014.
[2] J. F. Monserrat, G. Mange, V. Braun, H. Tullberg, G. Zimmermann, and Ö. Bulakci, "METIS research advances towards the 5G mobile and wireless system definition," EURASIP Journal on Wireless Communications and Networking, vol. 2015, no. 1, p. 53, Mar 2015

[3] D. Lopez-Perez, M. Ding, H. Claussen, and A. H. Jafari, "Towards $1 \mathrm{Gbps} / \mathrm{UE}$ in cellular systems: Understanding ultradense small cell deployments," IEEE Communications Surveys Tutorials, vol. 17, no. 4, pp. 2078-2101, Fourthquarter 2015.

[4] R. Baldemair, T. Irnich, K. Balachandran, E. Dahlman, G. Mildh, Y. Seln, S. Parkvall, M. Meyer, and A. Osseiran, "Ultra-dense networks in millimeter-wave frequencies," IEEE Communications Magazine, vol. 53, no. 1, pp. 202-208, January 2015.

[5] T. S. Rappaport, R. W. Heath Jr, R. C. Daniels, and J. N. Murdock, Millimeter wave wireless communications. Pearson Education, 2014.

[6] C. A. Balanis, Antenna Theory: Analysis and Design. WileyInterscience, 2005.

[7] J. G. Andrews, T. Bai, M. N. Kulkarni, A. Alkhateeb, A. K. Gupta, and R. W. Heath, "Modeling and analyzing millimeter wave cellular systems," IEEE Transactions on Communications, vol. 65, no. 1, pp. 403-430, Jan 2017.

[8] CST Computer Simulation Technology GmbH, Darmstadt, Germany, "CST-computer simulation technology microwave studio.” [Online]. Available: https://www.cst.com/products/cstmws

[9] M. R. Akdeniz, Y. Liu, M. K. Samimi, S. Sun, S. Rangan, T. S. Rappaport, and E. Erkip, "Millimeter wave channel modeling and cellular capacity evaluation," IEEE Journal on Selected Areas in Communications, vol. 32, no. 6, pp. 1164-1179, June 2014.

[10] A. Merwaday, R. Vannithamby, M. M. Rashid, Y. Zhang, C. Chen, and $\mathrm{X}$. Wu, "On the performance of directional communications in ultra-dense networks," in 2017 IEEE International Conference on Communications Workshops (ICC Workshops), May 2017, pp. 522-527.

[11] S. Gimenez, S. Roger, P. Baracca, D. Martín-Sacristán, J. F. Monserrat, V. Braun, and H. Halbauer, "Performance evaluation of analog beamforming with hardware impairments for mmw massive MIMO communication in an urban scenario," Sensors, vol. 16 , no. 10 , p. $1555,2016$.

[12] M. Rebato, L. Resteghini, C. Mazzucco, and M. Zorzi, "Study of realistic antenna patterns in $5 \mathrm{G}$ mmwave cellular scenarios," 2018. [Online]. Available: http://arxiv.org/abs/1802.01316

[13] 3rd Generation Partnership Project, "Evolved Universal Terrestrial Radio Access (E-UTRA) Further advancements for EUTRA physical layer aspects," 3GPP, Tech. Rep. 36.814, 2009, Release 9.

[14] P. Mogensen et al., "LTE capacity compared to the Shannon bound," in Vehicular Technology Conference, 2007. VTC2007Spring. IEEE 65th, April 2007, pp. 1234-1238. 